\title{
MICROPARTÍCULAS DE Ag/Ag 2 TIPO CORE-SHELL COMO SENSOR POTENCIOMÉTRICO PARA LA DETECCIÓN DE CIANURO
}

\author{
Renzo Olazo Quispe ${ }^{1}$, Adolfo La Rosa-Toro Gómez ${ }^{1 *}$
}

\begin{abstract}
RESUMEN
La síntesis de micropartículas de plata con película superficial de sulfuro de plata mezcladas con grafito en polvo ha permitido obtener un sensor electroquímico capaz de detectar cianuro con buena sensibilidad. Se caracterizó las micropartículas de plata y sulfuro de plata mediante voltametría cíclica (VC), Difracción de Rayos X (DRX), Fluorescencia de Rayos X (XRF), Microscopía de Barrido Electrónico(SEM) y Espectrometría de Energía Dispersiva de Rayos $\mathrm{X}(\mathrm{EDX})$. Se realizó ensayos potenciométricos para determinar el coeficiente de selectividad del sensor
\end{abstract}

Palabras clave: Micropartículas core-shell, $\mathrm{Ag} / \mathrm{Ag}_{2} \mathrm{~S}$, sensor de cianuro.

\section{MICROPARTICLES OF Ag/Ag $\mathrm{Ag}_{2}$ TYPE CORE-SHELL AS POTENCIOMETRIC SENSOR FOR DETECCION OF CYANIDE}

\begin{abstract}
The synthesis of silver microparticles with surface film of silver sulfide mixed with graphite powder has yielded an electrochemical sensor capable of detecting cyanide with good sensitivity. silver and silver sulfide microparticles was characterized by cyclic voltammetry (CV), X-ray diffraction (XRD), X-ray Fluorescence (XRF), Scanning Electron Microscopy (SEM) and Energy Dispersive Spectrometry X-ray (EDX). Potentiometric assays were performed to determine the selectivity coefficient of the sensor
\end{abstract}

Keywords: Microparticles core-shell, $\mathrm{Ag} / \mathrm{Ag}_{2} \mathrm{~S}$, cyanide sensor

\section{INTRODUCCIÓN}

El nivel de contaminación de las aguas es una preocupación permanente de las poblaciones cercanas a los asentamientos mineros y metalúrgicos, así como industrias en las zonas urbanas. El cianuro es uno de los principales contaminantes y forma parte de los efluentes de la industria galvánica, así como de la metalurgia de lixiviación de oro y plata ${ }^{1}$. Los ECA(DS N ${ }^{0}$ 002-2008 MINAM) para agua limitan el contenido de cianuro en el agua potable, con tratamiento químico, a un valor máximo de $0,022 \mathrm{ppm}^{2}$; es por ello que para el control del cumplimiento de los estándares en las aguas se requiere instrumentación sofisticada y de muy alto costo, entre ellas la cromatografía iónica, Raman y HPLC; una alternativa sin sacrificar exactitud y sensibilidad son los sensores fabricados en base a óxidos metálicos ${ }^{3}$. El presente trabajo se enfoca en la fabricación de un sensor potenciométrico con alta sensibilidad, capaz de medir la concentración de cianuro al nivel de las establecidas en las ECAs ${ }^{4}$. Los sensores

\footnotetext{
${ }^{1}$ Laboratorio de Electroquímica Aplicada. Facultad de Ciencia. Universidad Nacional de Ingeniería. Av. Túpac Amaru 210-Rimac/Lima 25-Perú. qirenzo17@gmail.com

Autor de correspondencia: toro@uni.edu.pe
} 
presentan la ventaja de su facilidad de bajo costo, preparación y fácil uso, siendo un método eficaz y eficiente.

\section{PARTE EXPERIMENTAL}

Reactivos. $\mathrm{AgNO}_{3},\left(\mathrm{NH}_{4}\right)_{2} \mathrm{~S}, \mathrm{NaOH}, \mathrm{HNO}_{3}$, alcohol polivinílico (PVP), etanol, $\mathrm{NaCN}$ y grafito conductor (GC).

Equipos. Potenciostato/Galvanostato, ORP, Difracción de Rayos X, Fluorescencia de Rayos X, Microscopía de Barrido Electrónico y Espectrometría de Rayos X.

Preparación de micropartículas de Ag. En un vaso de precipitación se preparó 0,2 M de $\mathrm{AgNO}_{3}$, se ajustó su $\mathrm{pH}$ hasta el valor de 7 añadiendo $\mathrm{NaOH} 0,1 \mathrm{M}$. En otro vaso precipitado se preparó $0,15 \mathrm{M}$ de ácido ascórbico y $4 \%$ de dispersante PVP (relativo a la masa de $\mathrm{AgNO}_{3}$ ). Ambas soluciones a un mismo volumen. Luego se vertió el primer vaso al segundo lentamente, formándose inmediatamente precipitado de polvo de plata La solución se filtró y se lavó con abundante agua ultrapura y etanol. El sólido se secó en una estufa a $60^{\circ} \mathrm{C}$ por 12 horas. ${ }^{6}$

Preparación de macropartículas de Ag encapsuladas con $\mathbf{A g}_{2}$ S. En un vaso de $200 \mathrm{~mL}$ se dispersa polvo de plata en una solución al $20 \%$ de sulfuro de amonio $\left(\mathrm{NH}_{4}\right)_{2} \mathrm{~S}$, mezclándolo cuidadosamente mediante agitador magnético, asegurando que todas las partículas se encuentren en contacto con la solución durante 8 minutos; posteriormente el polvo se separa mediante filtración y se enjuaga con agua ultrapura repetidas veces. Finalmente se seca a temperatura ambiente, obteniéndose micropartículas de plata- $\mathrm{Ag}_{2} \mathrm{~S}^{7}$ representándose como $\mathrm{Ag} / \mathrm{Ag}_{2} \mathrm{~S}$.

Limpieza del electrodo de carbón vítreo. La superficie del electrodo de carbón vítreo se pulió mecánicamente con papel abrasivo de 1000 y posteriormente alúmina de $0,3 \mu \mathrm{m}$ hasta obtener una superficie muy lisa y brillante; luego se enjuaga en acetona y se somete a ultrasonido en alcohol absoluto; posteriormente se sumergió en $\mathrm{HNO}_{3} 50 \%$ durante 1 minuto y en solución $1 \mathrm{M}$ de $\mathrm{NaOH}$, procediéndose a enjuagarlo en agua ultrapura de $18 \mathrm{M} \Omega . \mathrm{cm}$ de resistividad, procediendo a secarlo a temperatura ambiente. ${ }^{6}$

Construcción del sensor electroquímico. Se utilizó polvo de $\mathrm{Ag} / \mathrm{Ag}_{2} \mathrm{~S}$ como componente electroactivo con el cual se preparó los siguientes electrodos de estudio. ${ }^{8}$

a) Electrodo de $\mathrm{Ag} / \mathrm{Ag}_{2} \mathrm{~S}$ puro.

b) Electrodo de $\mathrm{Ag} / \mathrm{Ag}_{2} \mathrm{~S}-\mathrm{GC}$ mezcla de micropartículas de $\mathrm{Ag} / \mathrm{Ag}_{2} \mathrm{~S}$ con grafito conductor en las proporciónes $(1: 1),(1: 2)$ y $(1: 3)$.

En todos los casos se utilizó nujol como ligante. ${ }^{9}$

\section{RESULTADOS Y DISCUSIÓN \\ Cronopotenciometría de la conversión química de $\mathrm{Ag}_{2} \mathrm{~S}$ sobre la superficie de $\mathrm{Ag}$}

Se ha utilizado la técnica de cronopotenciometría para la determinación del tiempo de reacción de conversión química de la superficie de las micropartículas de plata en solución de $\left(\mathrm{NH}_{4}\right)_{2} \mathrm{~S}$ al $20 \%$ en volumen para la formación superficial de sulfuro de plata, $\mathrm{Ag}_{2} \mathrm{~S}$. El procedimiento experimental se realizó utilizando $0,1 \mathrm{M} \mathrm{Na}_{2} \mathrm{SO}_{4}$ como electrolito soporte; como electrodo de trabajo se utilizó un alambre de Ag, y calomel como electrodo de referencia. La figura 1 muestra la variación del potencial en función al tiempo de reacción; tiempo en el cual ocurre la formación de la película de $\mathrm{Ag}_{2} \mathrm{~S}$ sobre el alambre de Ag. Del 
gráfico se deduce que el cambio de potencial se encuentra relacionado al cambio químico que sucede en la superficie de la plata por reacción de la plata con el $\left(\mathrm{NH}_{4}\right)_{2} \mathrm{~S}$. A los 8 minutos de inmersión el potencial se estabiliza, pudiendo interpretarse como el punto final de la conversión química, habiéndose conseguido encapsular por completo la superficie de plata. El mismo procedimiento se aplicó a la micropartículas de plata para formar partículas de plata tipo core-shell.

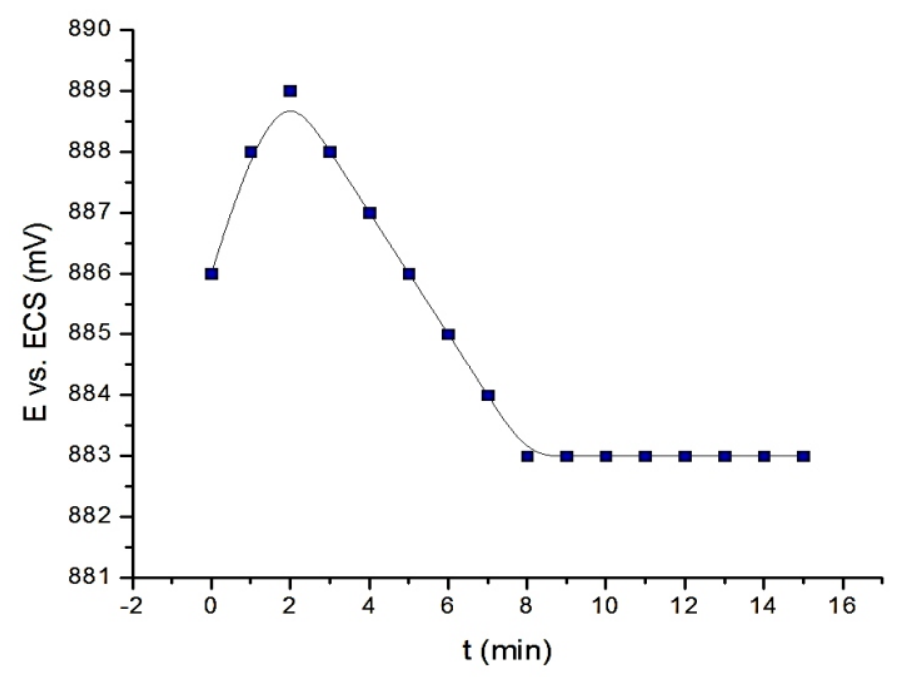

Figura 1. Respuestas del potencial frente al tiempo de las micropartículas de $\mathrm{Ag}$ sumergido en solución de $\left(\mathrm{NH}_{4}\right)_{2} \mathrm{~S} 20 \%$ y $0,1 \mathrm{M} \mathrm{Na} \mathrm{SO}_{4}$.

Caracterización voltamétrica de la mezcla de micropartículas de $\mathrm{Ag} / \mathrm{Ag}_{2} \mathrm{~S}$ y grafito conductor, $\mathrm{Ag} / \mathrm{Ag}_{2} \mathrm{~S}-\mathrm{GC}$, en solución $0,1 \mathrm{M}$ equimolar de $\mathrm{Fe}^{2+} / \mathrm{Fe}^{3+}$

La figura 2 muestra los voltagramas de las micropartículas de $\mathrm{Ag} / \mathrm{Ag}_{2} \mathrm{~S}$ mezcladas con grafito conductor en electrolito de $0,1 \mathrm{M}$ equimolar de $\mathrm{Fe}^{2+} / \mathrm{Fe}^{3+}$ empleando platino como contraelectrodo y calomel como electrodo de referencia. En el gráfico se observa que a potencial anódico de $0,05 \mathrm{~V}$ ocurre la oxidación de $\mathrm{Fe}^{2+} / \mathrm{Fe}^{3+}$ y a potencial de $-0,15 \mathrm{~V}$ se desarrolla la reducción de $\mathrm{Fe}^{3+} / \mathrm{Fe}^{2+}$. En el voltagrama, correspondiente a la mezcla $\mathrm{Ag} / \mathrm{Ag}_{2} \mathrm{~S}-$ GC (1:2) el perfil de óxido-reducción de la transición $\mathrm{Fe}^{2+} / \mathrm{Fe}^{3+}$ y viceversa, se muestra más definido que los electrodos preparados con las proporciones (1:1) y (1:3), debido a su mejor transferencia electrónica anódica-catódica con su entorno, seleccionándolo como la mejor mezcla para el sensor. 


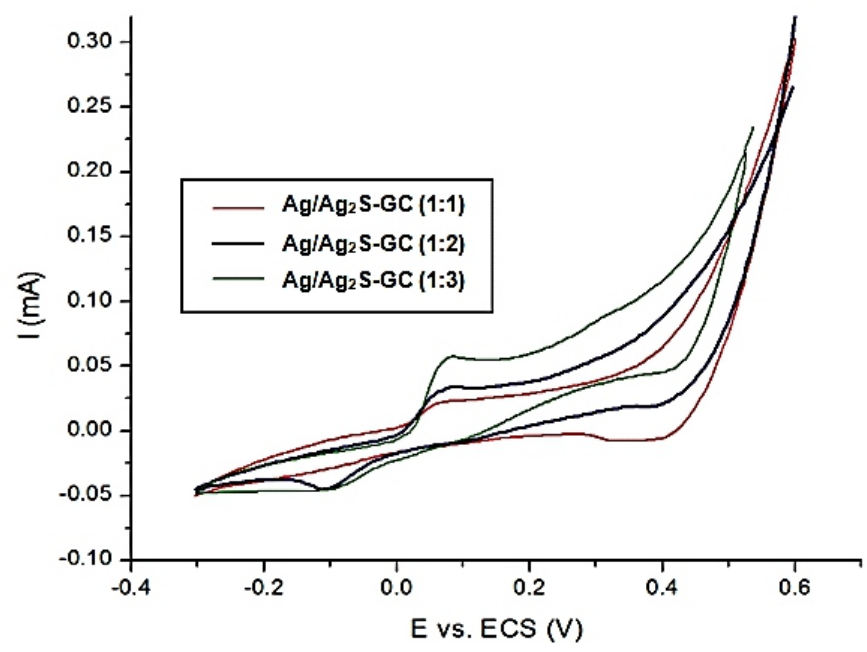

Figura 2: Voltametría cíclica de micropartículas de $\mathrm{Ag} / \mathrm{Ag}_{2} \mathrm{~S}-\mathrm{GC}(1: 1),(1: 2)$ y (1:3) en solución 0,1 equimolar de $\mathrm{Fe}^{2+} / \mathrm{Fe}^{3+}$ y $0,1 \mathrm{M} \mathrm{KNO}_{3}$. Velocidad de barrido $30 \mathrm{mV} / \mathrm{s}$.

En la figura 3, se muestra el comportamiento voltamétrico del electrodo $\mathrm{Ag} / \mathrm{Ag}_{2} \mathrm{~S}-\mathrm{GC}(1: 2)$ en solución $0,2 \mathrm{M}$ de $\mathrm{NaOH}$ y añadiendo progresivamente solución de cianuro de potasio. A potencial de $-0,6 \mathrm{~V}$ en sentido catódico se observa la formación de un pico de adsorción del ion $\mathrm{CN}^{-}$sobre la superficie del $\mathrm{Ag}_{2} \mathrm{~S}$ el cual presenta su correspondiente pico de desorción a potencial de $-0,45 \mathrm{~V}$ mostrando buena respuesta voltamétrica con el aumento de la concentración de ion $\mathrm{CN}^{-}$en la solución.

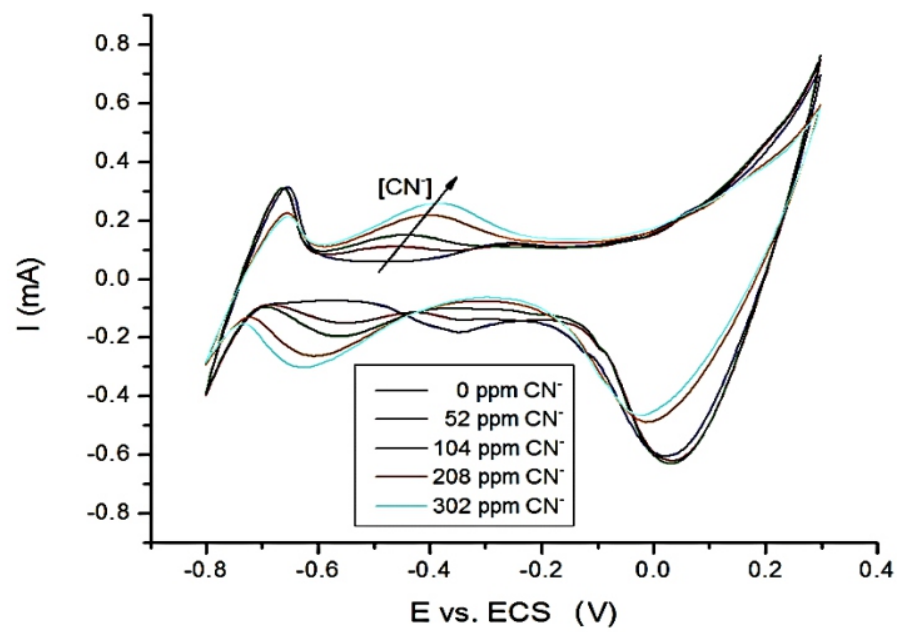

Figura 3. Voltametría cíclica de micropartículas de $\mathrm{Ag} / \mathrm{Ag}_{2} \mathrm{~S}$ en solución de $\mathrm{CN}^{-}$ con $0,2 \mathrm{M} \mathrm{NaOH}$. Velocidad de barrido $20 \mathrm{mV} / \mathrm{s}$. 


\section{Difracción de rayos X (DRX) para micropartículas $\mathrm{Ag}$ y $\mathrm{Ag} / \mathrm{Ag}_{2} \mathrm{~S}$}

En las figuras 4 y 5 se muestra los difractogramas de las micropartículas de $\mathrm{Ag} \mathrm{y} \mathrm{Ag} / \mathrm{Ag}_{2} \mathrm{~S}$, respectivamente. En la figura 5 se observa las líneas de difracción características de $\mathrm{Ag}, \mathrm{Ag}_{2} \mathrm{~S}$. El $\mathrm{Ag}_{2} \mathrm{~S}$ se presenta en 2 alótropos, acantita y argentita, respectivamente. La intensidad de la señal de $\mathrm{Ag}_{2} \mathrm{~S}$ es bastante menor comparado a la de $\mathrm{Ag}$, la que se encuentra relacionada a la abundancia de los mismos. En el gráfico in situ se observa las líneas de difracción magnificadas del $\mathrm{Ag}_{2} \mathrm{~S}$ en el rango de $2 \Theta$ de 30-37.

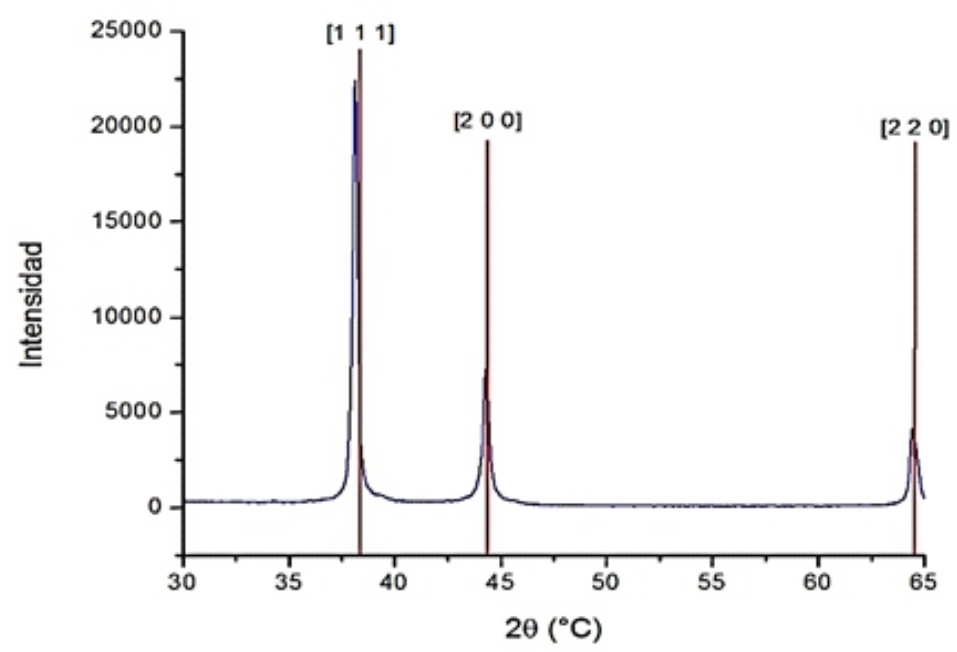

Figura 4. DRX de micropartículas de Ag y patrones de difracción de Ag.

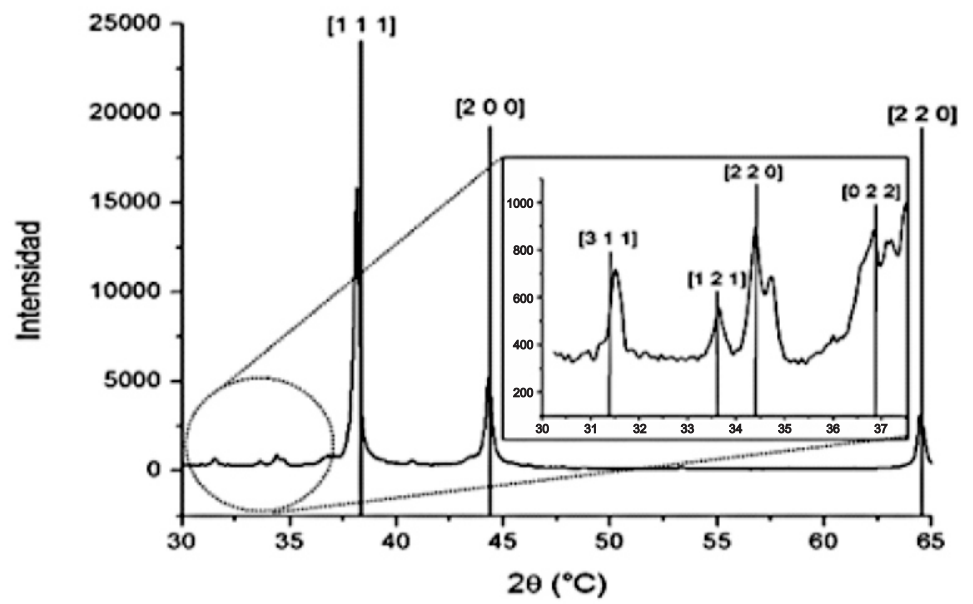

Figura 5. DRX de micropartículas de $\mathrm{Ag} / \mathrm{Ag}_{2} \mathrm{~S}$ y patrones de difracción de $\mathrm{Ag}$ y $\mathrm{Ag}_{2} \mathrm{~S}$. 
Fluorescencia de Rayos X (XRF) de micropartículas $\mathbf{A g}$ y Ag/ $/ \operatorname{Ag}_{2} \mathrm{~S}$

Se realizó el análisis químico de las muestras de micropartículas de $\mathrm{Ag}$ y $\mathrm{Ag} / \mathrm{Ag}_{2} \mathrm{~S}$ empleándose la técnica de Fluorescencia de Rayos X. En la figura 6 se muestra el espectro del análisis de la muestra de $\mathrm{Ag} / \mathrm{Ag}_{2} \mathrm{~S}$ y en la tabla 1 se presenta los resultados de la

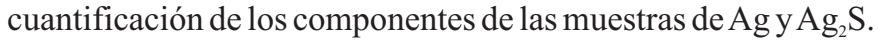

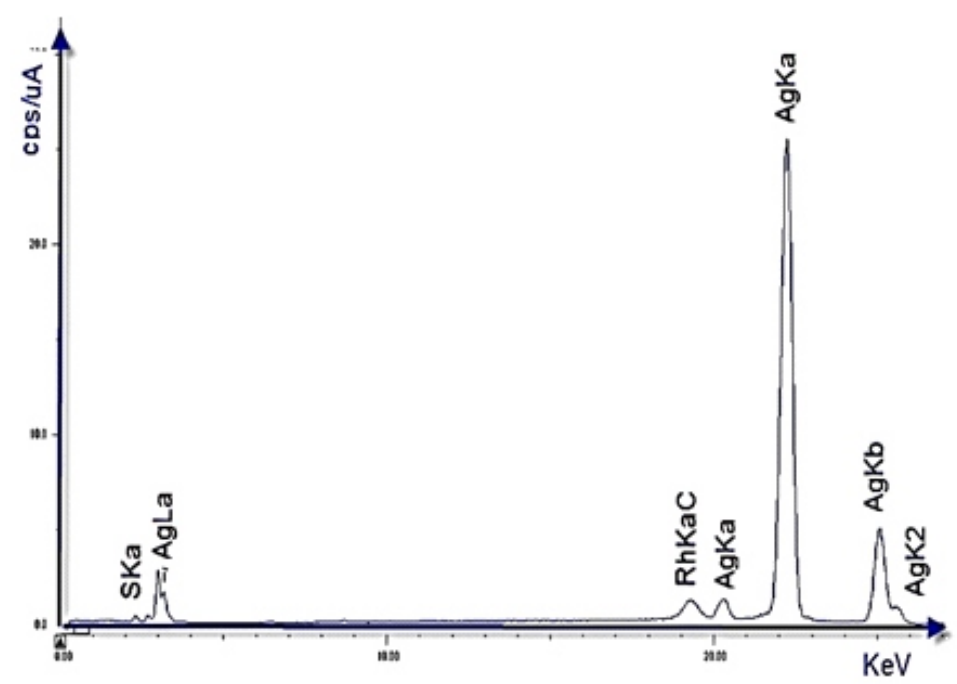

Figura 6. XRF de las micropartículas de $\mathrm{Ag} / \mathrm{Ag}_{2} \mathrm{~S}$.

Tabla 1. Porcentajes en masa de los elementos contenidos en muestra de $\mathrm{Ag}$ y $\mathrm{Ag} / \mathrm{Ag}_{2} \mathrm{~S}$.

\begin{tabular}{ccc}
\hline Elemento & $\begin{array}{c}\text { \% masa de } \\
\text { micropartículas de } \mathbf{A g}\end{array}$ & $\begin{array}{c}\text { \% masa de micropartículas } \\
\mathbf{d e ~} \mathbf{A g} / \mathbf{A g}_{2} \mathbf{S}\end{array}$ \\
$\mathrm{Ag}$ & 98,420 & 98,049 \\
$\mathrm{~S}$ & 0,549 & 1,5322 \\
$\mathrm{P}$ & 0,407 & 0,418 \\
$\mathrm{Fe}$ & 0,286 & - \\
$\mathrm{Si}$ & 0,176 & - \\
$\mathrm{Zn}$ & 0,162 & - \\
\hline
\end{tabular}

\section{Microscopía de barrido electrónico de micropartículas $\mathrm{Ag}$ y Ag/ $/ \mathrm{Ag}_{2} \mathrm{~S}$}

Las micropartículas de $\mathrm{Ag}$ y $\mathrm{Ag} / \mathrm{Ag}_{2} \mathrm{~S}$ fueron caracterizadas mediante la técnica Microscopia de Barrido Electrónico (SEM). Las imágenes SEM presentadas en la figura 7a y $7 \mathrm{~b}$ muestran una estructura conformada por micropartículas esféricas formando aglomeraciones distribuidas uniformemente. 


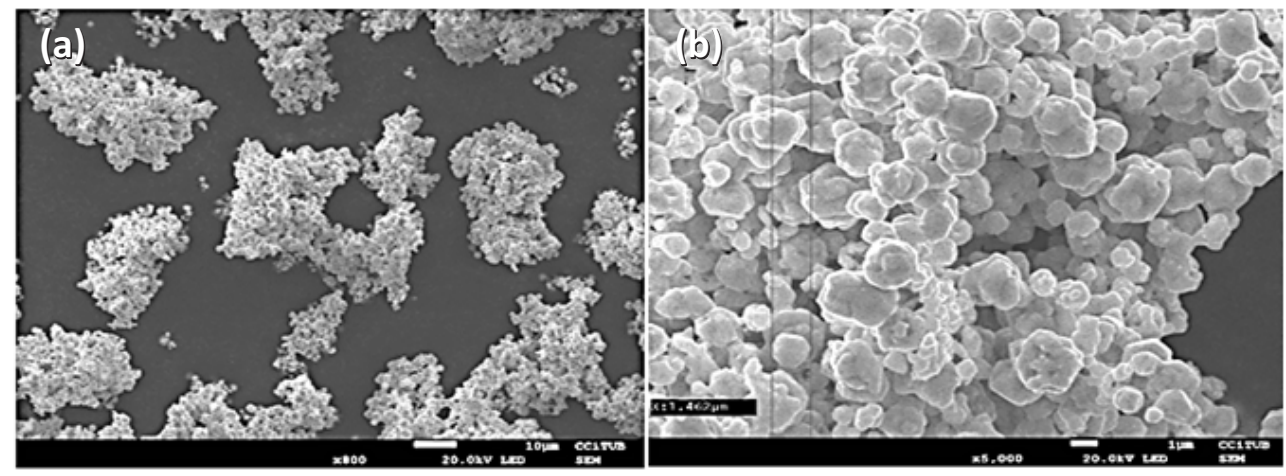

Figura 7. SEM para micropartícula de Ag a) 2000X b) 5000X.

En la figura 8 se presenta las imágenes SEM de la micropartículas de $\mathrm{Ag} / \mathrm{Ag}_{2} \mathrm{~S}$. En las amplificaciones x 5000 y x 10000 se puede observar superficies esféricas y homogéneas.

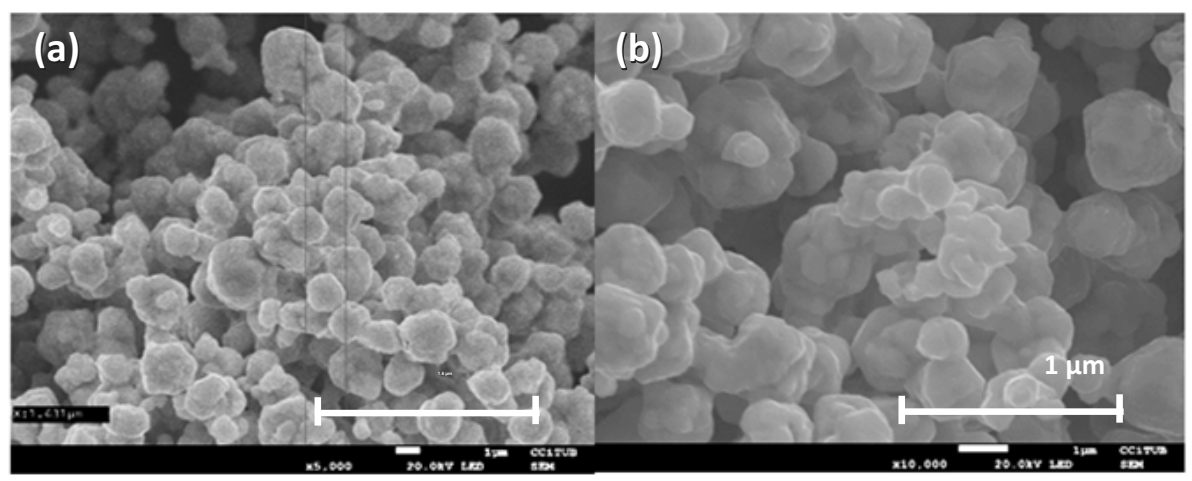

Figura 8. SEM de las micropartículas de $\mathrm{Ag} / \mathrm{Ag}_{2} \mathrm{~S}$ a) x5000 b) x10000.

El diámetro medio de las micropartículas fue determinado en las respectivas micrografías $\mathrm{SEM}$, siendo estas de 1,4 y 1,6 $\mu$ m para las micropartículas de $\mathrm{Ag}$ y $\mathrm{Ag} / \mathrm{Ag}_{2} \mathrm{~S}$, respectivamente.

\section{Espectrometría de dispersión de rayos $\mathrm{X}$ de las micropartículas $\mathrm{Ag}$ y $\mathbf{A g} / \mathrm{Ag}_{2} \mathrm{~S}$}

Se caracterizó las micropartículas de Ag mediante la técnica de Espectrometría de Dispersión de Rayos X (EDX). En la figura 9, se presenta el análisis EDX de micropartículas de Ag donde se observa la presencia de Ag y O. En la tabla 2, se presenta los porcentajes atómicos de los componentes. 


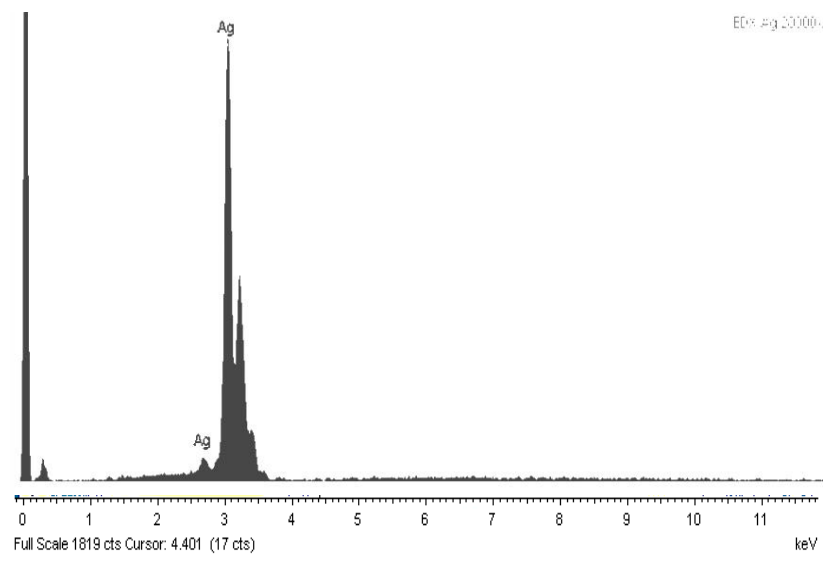

Tabla 2. Porcentaje atómico contenido en micropartículas de Ag.

\begin{tabular}{|c|c|}
\hline & \% atómico \\
\hline $\mathrm{Ag}$ & 93,1 \\
$\mathrm{O}$ & 6,9 \\
\hline
\end{tabular}

Figura 9. Análisis EDX de las micropartículas de Ag.

En la figura 10, se muestra el análisis EDX de las micropartículas de $\mathrm{Ag} / \mathrm{Ag}_{2} \mathrm{~S}$, mostrando la presencia de Ag, S y O. Los porcentajes atómicos de estos elementos se muestran en la tabla 3. La correlación $\mathrm{S} / \mathrm{Ag}$ en las micropartículas de $\mathrm{Ag} / \mathrm{Ag}_{2} \mathrm{~S}$ es de 0,062 , valor muy inferior a la relación estequiometría del componente $\mathrm{Ag}_{2} \mathrm{~S}$, igual a $\mathrm{S} / \mathrm{Ag}=0,5$. Por ello, se deduce que el $\mathrm{Ag}_{2} \mathrm{~S}$ se presenta como una película que encapsula las partículas esféricas de Ag presentando una estructura del tipo core-shell.

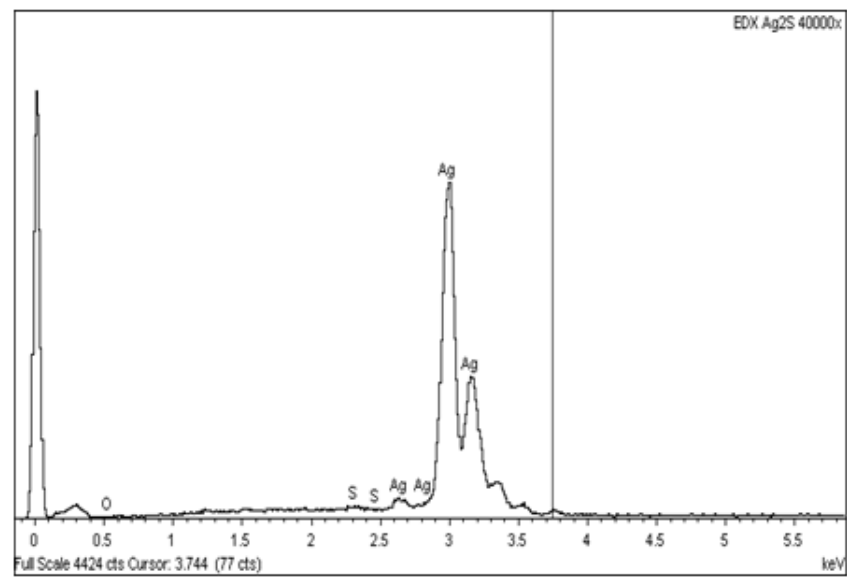

Tabla 3. Porcentaje atómico contenido en micropartículas de $\mathrm{Ag} / \mathrm{Ag}_{2} \mathrm{~S}$.

\begin{tabular}{|c|c|}
\hline & \% atómico \\
\hline $\mathrm{Ag}$ & 93,1 \\
$\mathrm{~S}$ & 3,55 \\
$\mathrm{O}$ & 6,9 \\
\hline
\end{tabular}

Figura 10. Análisis EDX de las micropartículas de $\mathrm{Ag} / \mathrm{Ag}_{2} \mathrm{~S}$. 


\section{Curva de calibración del sensor de micropartículas Ag/Ag $\mathbf{S}-\mathbf{G C}$}

En la figura 11, se observa las curvas de calibración del electrodo de micropartículas de $\mathrm{Ag} / \mathrm{Ag}_{2} \mathrm{~S}$ solo y mezcladas con grafito conductor en proporciones de 1:1, 1:2 y 1:3 en concentraciones controladas de ion cianuro.

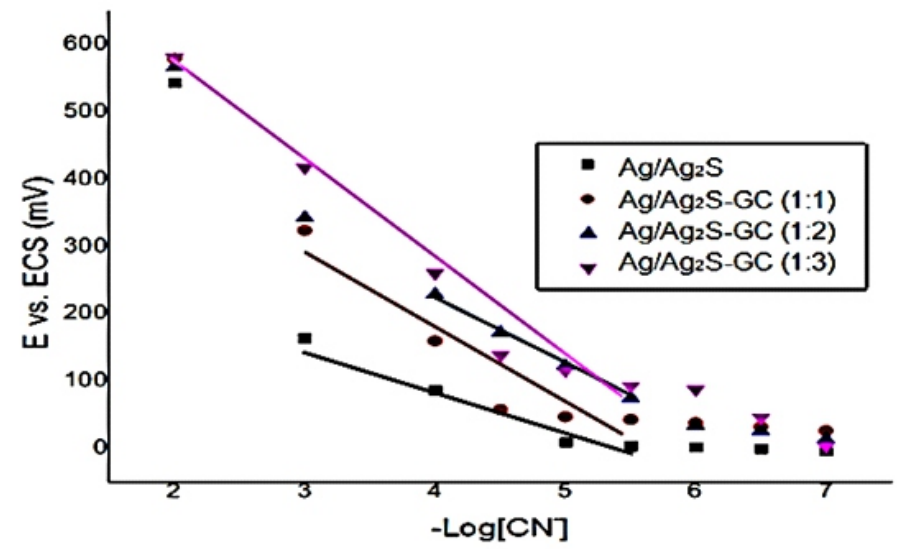

Figura 11: Curva de calibración del sensor de micropartículas de (a) $\mathrm{Ag} / \mathrm{Ag}_{2} \mathrm{~S}-\mathrm{GC}$ (1:1), (b) Ag/Ag 2 S-GC (1:2) y (c) Ag/Ag 2 S-GC (1:3)

En el gráfico, se puede observa una menor pendiente de respuesta al sensor fabricado de micropartículas de $\mathrm{Ag} / \mathrm{Ag}_{2} \mathrm{~S}$ sin mezcla, mejorando su respuesta con la mezcla de grafito conductor.

En la tabla 4, se presenta los resultados de la respuesta de los sensores preparados frente a la concentración del ión cianuro. De los datos obtenidos se puede observar que el sensor de micropartículas de $\mathrm{Ag} / \mathrm{Ag}_{2} \mathrm{~S}-\mathrm{GC}$ (1:2) presenta la mejor linealidad para el rango de concentración (-Log $\left.\left[\mathrm{CN}^{-}\right]\right)$de 4 a 6 , la mejor correlación de la pendiente igual a 0,9985 y el mejor límite de detección (LOD) igual a 0,020ppm.

Tabla 4. Valores de la ecuación y límite de detección para curvas de calibración de $\mathrm{Ag} / \mathrm{Ag}_{2} \mathrm{~S}-\mathrm{GC}$ (1:1), Ag/Ag ${ }_{2} \mathrm{~S}-\mathrm{GC}(1: 2), \mathrm{Ag} / \mathrm{Ag}_{2} \mathrm{~S}-\mathrm{GC}(1: 3)$ y Ag/ $\mathrm{Ag}_{2} \mathrm{~S}$.

\begin{tabular}{cccccc}
\hline & $\begin{array}{c}\text { Rango lineal } \\
(-\mathbf{L o g}[\mathbf{C N}])\end{array}$ & $\begin{array}{c}\text { Pendiente } \\
(\mathbf{m V} / \mathbf{d e c})\end{array}$ & $\begin{array}{c}\text { Intercepto } \\
(\mathbf{m V})\end{array}$ & $\mathbf{r}^{\mathbf{2}}$ & $\begin{array}{c}\text { LOD } \\
(\mathbf{p p m})\end{array}$ \\
$\mathrm{Ag} / \mathrm{Ag}_{2} \mathrm{~S}-\mathrm{GC}(1: 1)$ & 3 a 5,5 & $-106,31 \pm 22,32$ & $613,31 \pm 93,15$ & 0,9190 & 0,282 \\
$\mathrm{Ag} / \mathrm{Ag}_{2} \mathrm{~S}-\mathrm{GC}(1: 2)$ & 4 a 6 & $-92,70 \pm 4,29$ & $623,40 \pm 19,79$ & 09915 & 0,020 \\
$\mathrm{Ag} / \mathrm{Ag}_{2} \mathrm{~S}-\mathrm{GC}(1: 3)$ & 2 a 5,5 & $-147,65 \pm 12,82$ & $858,59 \pm 53,52$ & 0,9707 & 0,095 \\
$\mathrm{Ag} / \mathrm{Ag}_{2} \mathrm{~S}$ & 3 a 5,5 & $-66,98 \pm 7,71$ & $359,05 \pm 34,56$ & 0,9741 & 0,195 \\
\hline
\end{tabular}




\section{Reproducibilidad del sensor de micropartículas de $\mathrm{Ag} / \mathrm{Ag}_{2} \mathrm{~S}-\mathrm{GC}(1: 2)$}

Se realizó tres curvas de calibración del sensor de micropartículas de $\mathrm{Ag} / \mathrm{Ag}_{2} \mathrm{~S}-\mathrm{GC}$ (1:2) para evaluar su reproducibilidad. La figura 12 muestra que los potenciales son similares en cada curva de calibración. La tabla 5 muestra las pendientes y límites de detección mostrando una buena reproducibilidad para mediciones continuas de cianuro en agua.

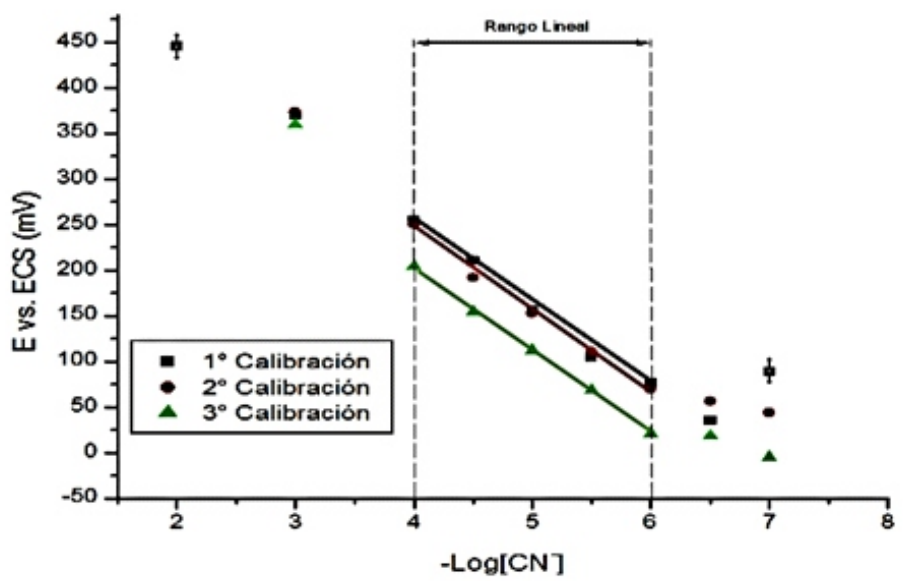

Figura 12. Ensayo de reproducibilidad del sensor de micropartículas $\mathrm{Ag} / \mathrm{Ag}_{2} \mathrm{~S}-\mathrm{GC}(1: 2)$.

En la tabla 5, se puede observar que para tres eventos de calibración de cianuro en el rango de concentración $\left(-\log \left[\mathrm{CN}^{-}\right]\right)$de $4-6$, los valores de regresión lineal es superior a 0,99 presentando un buen indicador de reproducibilidad para el sensor fabricado.

Tabla 5. Pendiente y límite de detección de micropartículas de Ag/ $\mathrm{Ag}_{2} \mathrm{~S}-\mathrm{GC}$ (1:2).

\begin{tabular}{cccccc}
\hline & $\begin{array}{c}\text { Rango lineal } \\
(-\mathbf{L o g}[\mathbf{C N}])\end{array}$ & $\begin{array}{c}\text { Pendiente } \\
(\mathbf{m V} / \mathbf{d e c})\end{array}$ & $\begin{array}{c}\text { Intercepto } \\
\mathbf{( m V )}\end{array}$ & $\mathbf{r}^{2}$ & $\begin{array}{c}\text { LOD } \\
\mathbf{( p p m )}\end{array}$ \\
$1^{\circ}$ Calibración & 4 a 6 & $-92,7 \pm 4.29$ & $623,4 \pm 19,79$ & 0,9150 & 0,020 \\
$2^{\circ}$ Calibración & 4 a 6 & $-88,5 \pm 6.12$ & $590,0 \pm 28,00$ & 0,9943 & 0,021 \\
$3^{\circ}$ Calibración & 4 a 6 & $-91,4 \pm 3.63$ & $610,0 \pm 16,10$ & 0,9991 & 0,021 \\
\hline
\end{tabular}

\section{Evaluación del sensor $\mathrm{Ag} / \mathrm{Ag}_{2} \mathrm{~S}-\mathrm{GC}(1: 2)$ frente a interferentes}

Se ha estudiado el comportamiento del sensor de micropartículas de $\mathrm{Ag} / \mathrm{Ag}_{2} \mathrm{~S}-\mathrm{GC}$ (1:2) frente a los interferentes en agua $\mathrm{Cl}^{-}, \mathrm{CO}_{3}{ }^{2-}$ y $\mathrm{S}^{2-}$; Los ensayos fueron realizados evaluando los electrodos a concentración definida de interferente y calibrando el sensor frente a la concentración de cianuro. 
En la figura 13, se muestra la variación de la curva de calibración del sensor de micropartículas de $\mathrm{Ag} / \mathrm{Ag}_{2} \mathrm{~S}-\mathrm{GC}(1: 2)$ en presencia del interferente $\mathrm{CO}_{3}{ }^{2-}$. La tabla 6 muestra que a $0,1 \mathrm{ppm}$ y $1 \mathrm{ppm} \mathrm{CO}_{3}{ }^{2-}$ se presenta un descenso de la pendiente conservando la linealidad. Sin embargo, a $5 \mathrm{ppm} \mathrm{CO}_{3}^{2-}$ se afecta significativamente la linealidad.

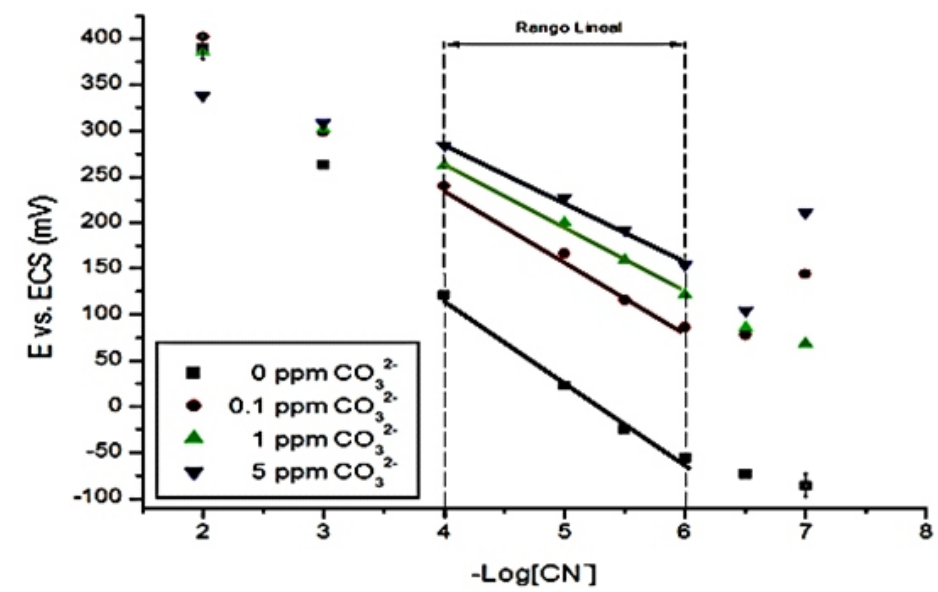

Figura 13. Curva de calibración del sensor de micropartículas de $\mathrm{Ag} / \mathrm{Ag}_{2} \mathrm{~S}-\mathrm{GC}(1: 2)$ en presencia de $\mathrm{CO}_{3}{ }^{2-}$.

Tabla 6. Variación de pendiente de micropartículas de $\mathrm{Ag} / \mathrm{Ag}_{2} \mathrm{~S}-\mathrm{GC}(1: 2)$ en presencia de $\mathrm{CO}_{3}^{2-}$.

\begin{tabular}{lcccc}
\hline $\begin{array}{c}\text { Interferente } \\
\text { carbonato }\end{array}$ & $\begin{array}{c}\text { Rango lineal } \\
(-\mathbf{L o g}[\mathbf{C N}])\end{array}$ & $\begin{array}{c}\text { Pendiente } \\
(\mathbf{m V} / \mathbf{d e c})\end{array}$ & $\mathbf{r}^{2}$ & $\begin{array}{c}\text { Variación en } \\
\text { pendiente }\end{array}$ \\
0 ppm & 4 a 6 & $-90,229$ & 0,9928 & - \\
$0,1 \mathrm{ppm}$ & 4 a 6 & $-78,629$ & 0,9950 & $12,86 \%$ \\
$1 \mathrm{ppm}$ & 4 a 6 & $-69,943$ & 0,9960 & $22,48 \%$ \\
$5 \mathrm{ppm}$ & 4 a 6 & $-64,514$ & 0,9947 & $28,50 \%$ \\
\hline
\end{tabular}

En la figura 14 y tabla 7, se muestra los resultados del estudio de interferencia del ion sulfuro. De acuerdo a los resultados del gráfico, se observa que a concentración de $0,1 \mathrm{ppm}$ de $\mathrm{S}^{2-}$ el rango de sensibilidad se acorta variando a $\left(-\log \left[\mathrm{CN}^{-}\right]\right)$de 3 a 5,5 A concentración de 1 ppm la linealidad y pendiente se afectan significativamente. 


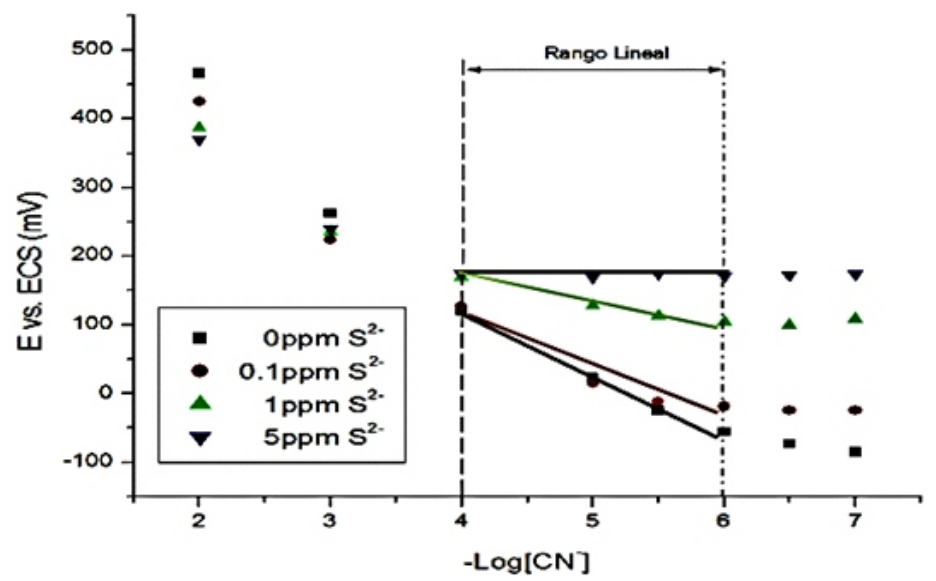

Figura 14. Curva de calibración del sensor de micropartículas de $\mathrm{Ag} / \mathrm{Ag}_{2} \mathrm{~S}-\mathrm{GC}$ (1:2) en presencia de $\mathrm{S}^{2-}$.

Tabla 7. Variación de pendiente para micropartículas de $\mathrm{Ag} / \mathrm{Ag}_{2} \mathrm{~S}-\mathrm{GC}(1: 2)$ en presencia de $\mathrm{S}^{2-}$.

\begin{tabular}{lcccc}
\hline $\begin{array}{c}\text { Interferente } \\
\text { sulfuro }\end{array}$ & $\begin{array}{c}\text { Rango lineal } \\
\left(-\mathbf{L o g}\left[\mathbf{C N}^{-}\right]\right)\end{array}$ & $\begin{array}{c}\text { Pendiente } \\
(\mathbf{m V} / \mathbf{d e c})\end{array}$ & $\mathbf{r}^{\mathbf{2}}$ & $\begin{array}{c}\text { Variación en } \\
\text { pendiente }\end{array}$ \\
0 ppm & 4 a 6 & $-90,229$ & 0,9928 & - \\
$0,1 \mathrm{ppm}$ & 4 a 6 & $-75,829$ & 0,9129 & $15,96 \%$ \\
$1 \mathrm{ppm}$ & 4 a 6 & $-23,086$ & 0,9930 & $74,41 \%$ \\
$5 \mathrm{ppm}$ & 4 a 6 & $-1,257$ & 0,1280 & $98,60 \%$ \\
\hline
\end{tabular}

En la tabla 8 se muestra los resultados de las evaluaciones del interferente $\mathrm{Cl}^{-}$en la cual se observa que la pendiente sufre variaciones con respecto al aumento de contenido del ión $\mathrm{Cl}^{-}$en la solución.

Tabla 8. Variación de pendiente de micropartículas de $\mathrm{Ag} / \mathrm{Ag}_{2} \mathrm{~S}-\mathrm{GC}(1: 2)$ en presencia de $\mathrm{Cl}^{-}$.

\begin{tabular}{lcccc}
\hline $\begin{array}{c}\text { Interferente } \\
\text { cloruro }\end{array}$ & $\begin{array}{c}\text { Rango lineal } \\
\left(-\mathbf{L o g}\left[\mathbf{C N}^{-}\right]\right)\end{array}$ & $\begin{array}{c}\text { Pendiente } \\
(\mathbf{m V} / \mathbf{d e c})\end{array}$ & $\mathbf{r}^{2}$ & $\begin{array}{c}\text { Variación en } \\
\text { pendiente }\end{array}$ \\
0 ppm & 4 a 6 & $-90,229$ & 0,9928 & - \\
$0,1 \mathrm{ppm}$ & 4 a 6 & $-83,829$ & 0,9888 & $7,10 \%$ \\
$1 \mathrm{ppm}$ & 4 a 6 & $-79,829$ & 0,9812 & $11,52 \%$ \\
$5 \mathrm{ppm}$ & 4 a 6 & $-74,229$ & 0,9713 & $17,73 \%$ \\
\hline
\end{tabular}




\section{Coeficiente de selectividad}

Con el propósito de establecer cuantitativamente el efecto de los interferentes $\mathrm{Cl}^{-}, \mathrm{CO}_{3}{ }^{2-}$ y $\mathrm{S}^{2-}$ en la medida del ion cianuro se aplicó el modelo matemático de soluciones constantes para la determinación del coeficiente de selección del sensor de micropartículas de $\mathrm{Ag} / \mathrm{Ag}_{2} \mathrm{~S}-\mathrm{GC}$ (1:2) con los correspondientes interferentes. En la tabla 9, se muestra que el ion sulfuro es una fuerte interferente en la mediciones potenciométricas de cianuro.

Tabla 9. Coeficiente de selectividad del sensor de micropartículas de $\mathrm{Ag} / \mathrm{Ag}_{2} \mathrm{~S}-\mathrm{GC}(1: 2)$.

\begin{tabular}{lr}
\hline Interferentes & $\mathbf{K}_{\mathbf{C N}^{-}}^{\text {pot }} \mathbf{I n t}$ \\
Sulfuro & 1,04 \\
Cloruro & 0,39 \\
Carbonato & 0,07 \\
\hline
\end{tabular}

\section{CONCLUSIONES}

Se preparó micropartículas de Ag mediante reducción química, lo que fue tratado en solución de sulfuro de amonio para la conversión química de la superficie, formando micropartículas de $\mathrm{Ag} / \mathrm{Ag}_{2} \mathrm{~S}$ tipo core-shell. El electrodo fue caracterizado por voltametría cíclica y DRX, confirmándose la formación de $\mathrm{Ag}_{2} \mathrm{~S}$ sobre la superficie de las micropartículas de $\mathrm{Ag}$. $\mathrm{La}$ forma esférica de las micropartículas y el diámetro medio fue determinado mediante análisis SEM. La composición química fue analizada mediante Fluorescencia de Rayos X y EDX. La evaluación del comportamiento rédox de los electrodos preparados indican que el sensor de micropartículas de $\mathrm{Ag} / \mathrm{Ag}_{2} \mathrm{~S}-\mathrm{GC}$ (1:2) presenta la mejor sensibilidad, alcanzando linealidad para mediciones de $1 \times 10^{-6} \mathrm{M}$ de $\mathrm{CN}^{-}$. La evaluación de iones interferentes indican que la sensibilidad al cianuro es afectado en el siguiente orden: $\mathrm{S}^{2-}>\mathrm{Cl}^{-}>\mathrm{CO}_{3}^{2-}$.

\section{AGRADECIMIENTO}

Los autores agradecen al Instituto de Investigación de la Universidad Nacional de Ingeniería (IGI- 2012) y al CONCYTEC-proyecto especial del PROCYT 2012, por los recursos económicos aportados para la realización del presente proyecto.

\section{BIBLIOGRAFÍA}

1. Logsdon, Mark; Hagelstein, Karen; Mudder, Terry. El manejo del cianuro en la extracción de oro. Consejo Internaciónal de Metales y Medio Ambiente. 2001: 7-10.

2. Estándares nacionales de calidad ambiental para agua. http://intranet2.minem.gob.pe/Web/archivos/dgaae/legislación/DS002-2008.pdf

3. A. La Rosa-Toro, R. Berenguer, C. Quijada, F. Montilla, E. Morallon, and J. L. Vázquez, Preparation and Characterization of Copper-Doped Cobalt Oxide Electrodes, J. Phys. Chem. B 2006; 110: 24021-24029

4. Cano, Manuel. Diseño y Aplicación de Sensores Electroquímicos basados en Moléculas Orgánicas Conductoras. Departamento de Química Física y Termodinámica Aplicada. [Tesis Doctoral]. Universidad de Córdoba: España. 2008: 19- 22. 
5. Cuervo, G. Efecto de la variación de la composición y del espesor de la membrana selectiva sobre el comportamiento de electrodos selectivos de contacto sólido para $\mathrm{Pb}^{2+}$. XXV Congreso de la Sociedad Mexicana de Electroquímica. México. 2010.

6. Guiquan Guo. Preparation and dispersive mechanism of highly dispersive ultrafine silver powder. Applied Surface Science. 2010; 256: 6683-6687

7. Dobenik, D. A silver/silver sulphide selective electrode prepared by means of chemical treatment of silver wire. Acta Chim. Slov. 1998; 45(3): 209-216.

8. Maria Gismera. Desarrollo de Nuevos Sensores Potenciométricos para Metales basados en Receptores con grupos tio y ditio. Universidad Autónoma de Madrid: España. 2006: 3-7.

9. Lázaro, Osvaldo. Furoiltioureas: Naturaleza de sus complejos con $\mathrm{CdCl}_{2}$ y $\mathrm{HgCl}_{2}$ y su utilización en sensores electroquímicos. [Tesis Doctoral] Universidad de Cádiz. España. 2006: 25-26. 